\title{
Rigidity in synkinetic molecular monolayers of functional lipids containing Ångström gaps
}

\author{
Jürgen-Hinrich Fuhrhop*, Laurent Ruhlmann, Christian Messerschmidt, Werner \\ Fudickar, Jörg Zimmermann and Beate Röder \\ Institut für Organische Chemie, der Freien Universität Berlin, Takustraße 3, D-14195 Berlin, Germany
}

\begin{abstract}
Bolaamphiphiles ("bola") are compounds with a hydrophobic core and hydrophilic head groups at both ends. The head groups as well as the hydrophobic core may be functional in nonsymmetric arrangements. One end group may, for example, be bound to a solid subphase, the other may dissolve and react with solutes in the bulk water phase. The hydrophobic core may contain reactive $\mathrm{C}=\mathrm{C}$ double bonds. If a monolayer membrane is formed from the bolaamphiphile, only the outer head groups and the double bonds at the edges of the membrane can react with polar reagents in the bulk water phase, provided the membrane is rigid enough. Rigidity may be achieved either by rigid hydrophobic skeletons (carotenes, steroids, porphyrins) or by two hydrogen bond chains between secondary amide groups within an oligomethylene chain. Rigid monolayers have so far been used to prepare water-free, dry vesicle membranes, to protect solid surfaces from aggressive reagents by an ultrathin lipid layer and, most important, to construct functionalized gaps in membranes on solid surfaces. The latter are thought to contain water molecules, which do not exchange with the bulk water ("hydrophobic water") and may bind watersoluble molecules.
\end{abstract}

\section{INTRODUCTION}

Bolas with two different head groups at the ends of oligomethylene chains or oligomethylene macrocycles provide membranes with a specific type of reactivity and stereochemistry. It has been possible in several cases to dissolve them in water under given conditions and to convert them afterwards quantitatively and reversibly to polymeric molecular assemblies. Well-known examples are the one-sided precipitation of bolas to give vesicles by a change of $\mathrm{pH}$ (ref. 1) or ammonium-counterions $\left(\mathrm{Br}^{-} \rightarrow \mathrm{ClO}_{4}{ }^{-}\right.$; ref. 2$)$ and the self-assembly of $\alpha$-mercapto- $\omega$-carboxylates on gold surfaces (ref. 3). One obtains, in general, spherical or planar monolayers with a totally unsymmetrical arrangement of the head groups. In vesicles, all large head groups may be on the larger outer surface (refs. 1 and 4), and all small head groups on the smaller inner surface. In self-assembled monolayers, one may position electron-accepting quinones on the gold surface or inside the membrane and corresponding donors on the outside. Many such asymmetric donor-acceptor systems have been produced, both in bulk, vesicular solution and on solid surfaces, but attempted lightinduced charge separation has not become useful. Charge recombination in such fluid membranes is invariably at least as fast as charge separation (ref. 5).

Furthermore, the flexibility of alkyl chains connecting the head groups not only causes fluidity in vesicle membranes, but also leads to a massive disordering of the edges of monolayer domains on solid surfaces (Fig.1; ref. 6): It thus becomes difficult to produce stable molecular gaps of Ångström size in molecular monolayers by a stepwise deposition of flat-lying molecules and up-standing bolas.

To regulate distances between reactive clusters and produce membrane gaps of defined size, one has to replace the fluid membrane structures by more rigid ones. An immediate consequence is that the nondirectional repulsive hydration forces between the head group acting in spherical micelles and vesicles must be replaced by directional binding forces. Instead of "self-organization" (ref. 7), non-covalent synthesis or synkinesis (ref. 8) must be applied. Each molecule of the molecular assembly is considered now as a fitting synkinon, which binds in all parts to its neighbors in the polymeric molecular assembly. Synkinesis must, however, not lead to three-dimensional crystals. Crystallisation can be prevented either by curvature of the molecular assemblies, which leads either to undulating vesicle or molecular fibers.

* Lecture presented at the 1st International Conference on Supramolecular Science and Technology, Zakopane, Poland,

27 September-3 October 1998.

Other presentations are published in this issue, pp. 2337-2408. 

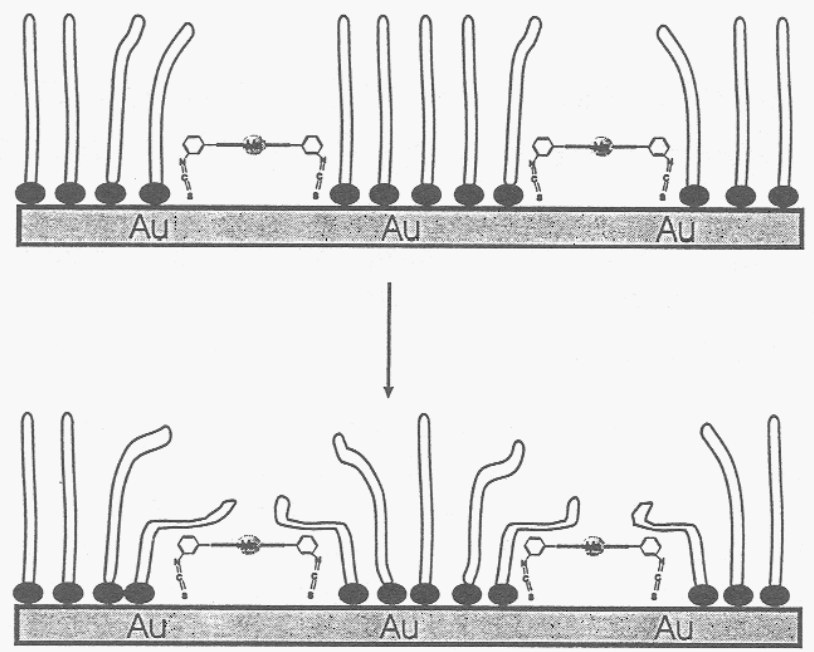
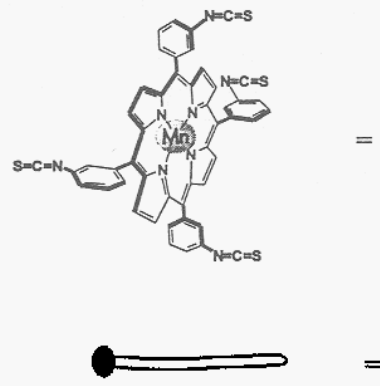

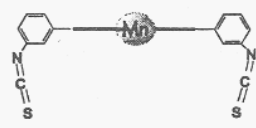

$\mathrm{C}_{18} \mathrm{SH}$

Fig. 1 (a) A one-dimensional model of an ideal molecular gap of a liquid membrane on a solid surface and (b) a realistic model with statistical lipid conformations at the edges of the membrane. Here a manganese porphyrinate, which is covalently bound to the gold subphase, is used as a matrix.

Four different hydrophobic skeletons have been applied to synkinesize two-dimensional crystalline monolayers from bolas:

(i) diphenyl or diacetylene units within flexible oligomethylene chains. Appropriate bola-dianions and dications have been used to assemble 35-meric multilayers. These are slightly inhomogenous, but easy to assemble (ref. 9).

(ii) the polyene chain of bixin, which allows the formation of isolable vesicles (ref. 15).

(iii) two secondary amide groups are forming hydrogen bonds, which gives impermeable and rigid monolayers (ref. 17).

(iv) porphyrin amphiphiles and bolaamphiphiles have been used to produce micellar fibers (refs. 10 and 11), vesicular tubules (ref. 12) and leaflets (ref. 13).

In the following, we discuss our own experimental results concerning rigid monolayers of types (ii)-(iv).

\section{POLYENE BOLAAMPHIPHILES}

Bixin is a commercially accessible polyene-bola with one free carboxylate and one methylester end group. In surface monolayers on water, it lies almost parallel to the surface at low pressure and changes to an upright position at higher pressures (ref. 14). Upon sonication, vesicles are formed, but they tend to aggregate and do not survive drying. The situation changes, if four bixin molecules are bound to a porphyrin. The resulting mixture of four atropisomeric tetraesters gives uniform vesicles with membranes of monolayer thickness $(4.9 \mathrm{~nm}$ ) (Fig. 2a; ref. 15). These vesicles are completly stable in concentrated salt 

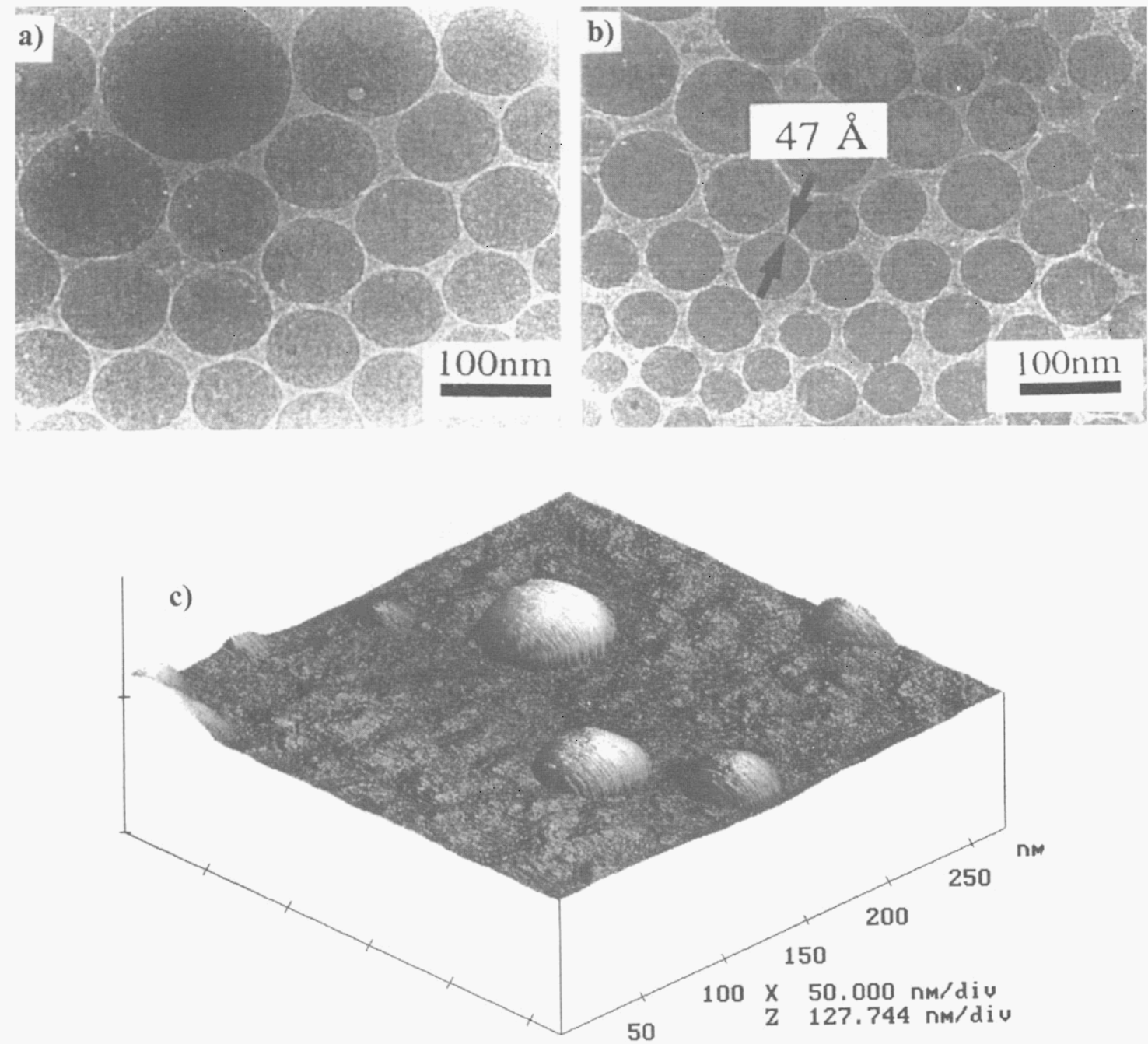

Fig. 2 Transmission electron micrograph of the vesicles made of the tetra-[bixinato-o-phenol]porphyrin (a) after and (b) before polymerization. (c) AFM of the polymeric vesicle membrane on graphite.

solutions, which indicates a porous membrane. On hydrophobic solid surfaces they survive drying. Atomic force field microscopy shows a perfect sphere on graphite. On more hydrophilic surfaces, slow flattening of the sphere occurs. Irradiation with visible light leads to a porphyrin-sensitized polymerization of the polyene. The vesicles now consist of a single molecule as is shown by suspension in pure ethanol. No monomers are dissolved, nor do holes appear in the vesicle membrane (Fig. 2c). AFM shows that the polymeric vesicle also survives drying.

This polymer is the first monomolecular sphere with a large internal volume. More interesting is, however, the polymerization of the corresponding surface monolayer on water. Upon irradiation with visible light the molecular area of the tetrabixinatoporphyrin shrinks from $270 \mathrm{~nm}^{2}$ to $180 \mathrm{~nm}^{2}$ indicating polymerization. The bixin chromophores are completely bleached (ref. 15). It is thought that the whole macroscopic monolayer is now photo cross-linked to a single molecule. After transfer onto a silicon surface, however, one finds large swollen blisters with a height of about $15 \mathrm{~nm}$. After drying, a $4 \mathrm{~nm}$ structured bilayer is formed. Irradiation obviously not only leads to lateral polymerization within the surface monolayer, but also to the partial new packing of swellable bilayers (Fig. 3; ref. 16).

The structured monolayer of a totally water-insoluble, bolaamphiphilic monolayer is in principle a useful means to activate smooth organic or inorganic surfaces. Gold, silicon, and polyacrylnitrile surfaces, for example, may be converted to poly(carboxylate) surfaces without any need to consider the individual surface reactivity. In order to be useful, however, the "monomolecular cloth" must be made as smooth and 


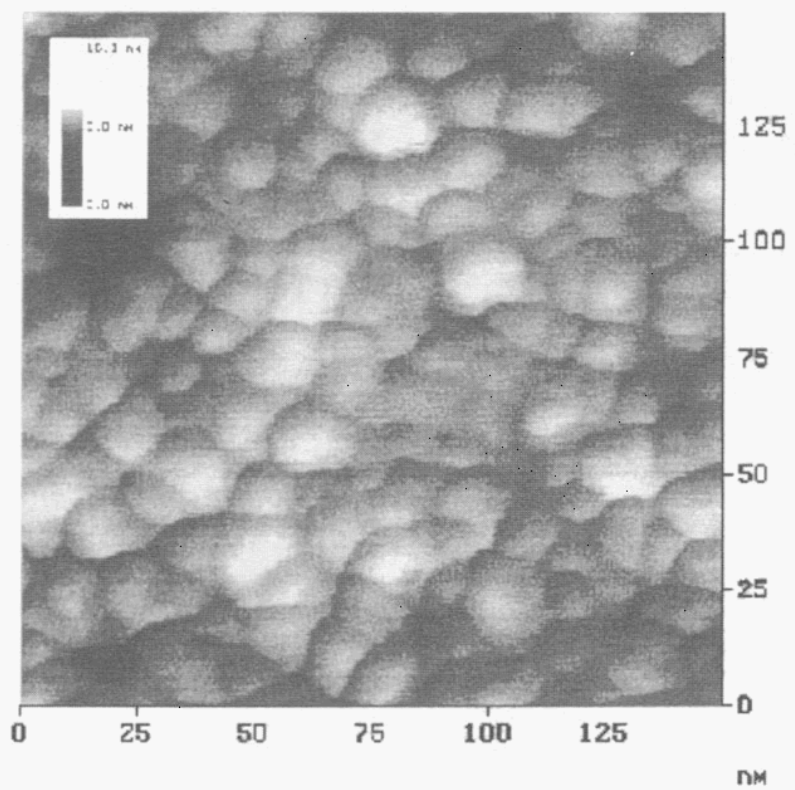

Fig. 3 AFM picture of a polymerized Langmuir-Blodgett type monolayer of the tetrabixinatoporphyrin on mica. The typical $15 \mathrm{~nm}$ layer shrinks after drying to $4.7 \mathrm{~nm}$.

simple as the subphase, which it covers. This has been achieved with more simple bixin amides (unpublished results).

\section{DIAMIDE LIPIDS}

Bolas with two $\alpha, \omega$-benzoylazide head groups are water-stable and form stable, upright monolayers on water. LB-films on polyacrylonitrile surfaces are crystalline and diffract electrons. Upon treatment with gaseous methylamine, only the outer benzoylazide groups are quantitatively converted to benzoylamide functions, whereas the inner group does not react in measurable quantities for several hours. The crystalline two-dimensional monolayer is impermeable to methylamine. If there is only one secondary amide group in the bola chain and the monolayer is not crystalline, no such in-outside differentiation is observed (ref. 17).

$\alpha$-Mercapto- $\omega$-acetamido bolas with primary amide groups form crystalline monolayers, if the connecting methylene-chain is even-numbered. Odd-numbered connecting chains give fluid membranes. The tilting of the monolayer allows two parallel amide hydrogen bond chains only if both amide groups face each other in the same way. With odd-numbered connecting links, the second carbonyl and amine groups point towards neighboring methylene groups (Fig. 4; ref. 18). The material properties of synkinetic monolayers thus heavily depend on an appropriate stereochemistry of the monomers.

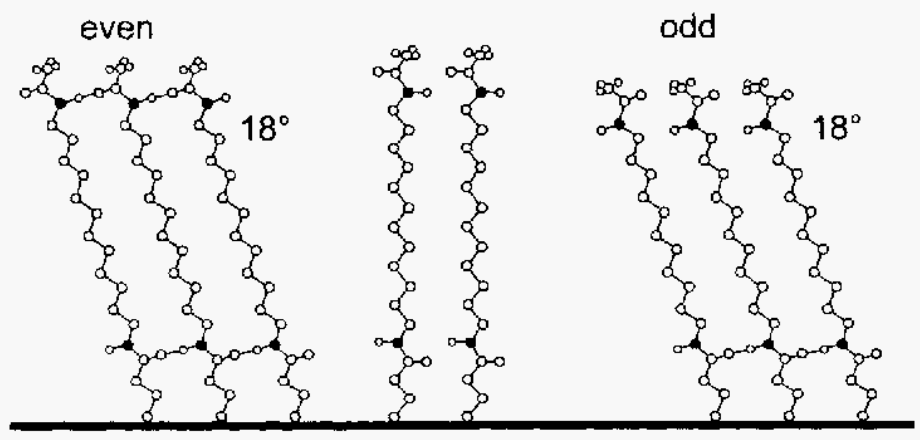

Fig. 4 Odd-even effect in tilted diamide monolayers. The even-numbered monolayer is crystalline and impermeable; the odd-numbered homologue fluid. A non-tilted monolayer does not show such an effect. 


\section{GAPS IN FLUID MONOLAYERS AND IMMOBILE WATER}

A steroid with an axial SH-group in the molecule's center and octadecanethiol were subsequently bound to a gold surface. This was possible, because the bulky steroid covering the AuS bound prevented substitution by $\mathrm{C}_{18} \mathrm{SH}$. The steroid- $\mathrm{C}_{18} \mathrm{SH}$ covered gold electrode still allowed electron transport from ferric cyanide ions to the gold surface as was shown by cyclic voltammetry. Upon addition of 1,2-trans-cyclohexanediol, however, the current was almost totally blocked. The effect was persistent for many days. Bulk water did not remove the diol with two equatorial $\mathrm{OH}$-groups. This was only released upon addition of ethanol or acids, which destroyed the water-cyclohexanediol supramolecular structure within the hydrophobic gaps. 1,2-cis-Cyclohexanediol had hardly any effect on the ferricyanide response; D-glucose and L-rhamnose were about a fifth as effective as trans-1,2-cyclohexanediol. The rational behind these observations is presumably the total immobilization of the surface water within the $7 \AA$-wide hydrophobic membrane gaps above the steroids (ref. 19). Only about four water molecules or one 1,2-trans-cyclohexane diol molecule are needed to bridge the gap, which has the width of a steroid. Since about 2-3 water molecules are thought to be immobilized on hydrophobic surfaces, all water molecules within the hydrophobic gap should be immobile. This effect is, however, not sufficient to block the transport of ferricyanide ions to the steroid surface and electron tunneling to the gold electrode. Only upon co-crystallization with fitting and rigid cyclohexane-derivatives does the "ice-cube" within the hydrophobic gap becomes an solid barrier to ion transport (Fig. 5).

The above results indicate that the hydrophobic effect alone enforces already quite regular pores in the membrane above the steroids. The oligomethylene chains on the edges of the gold-bound steroids do not cover up the gap, and formation of ordered assemblies is possible. Surprisingly, disturbances by tipping over of the edge amphiphiles as illustrated in Fig. 1 must not be very important.
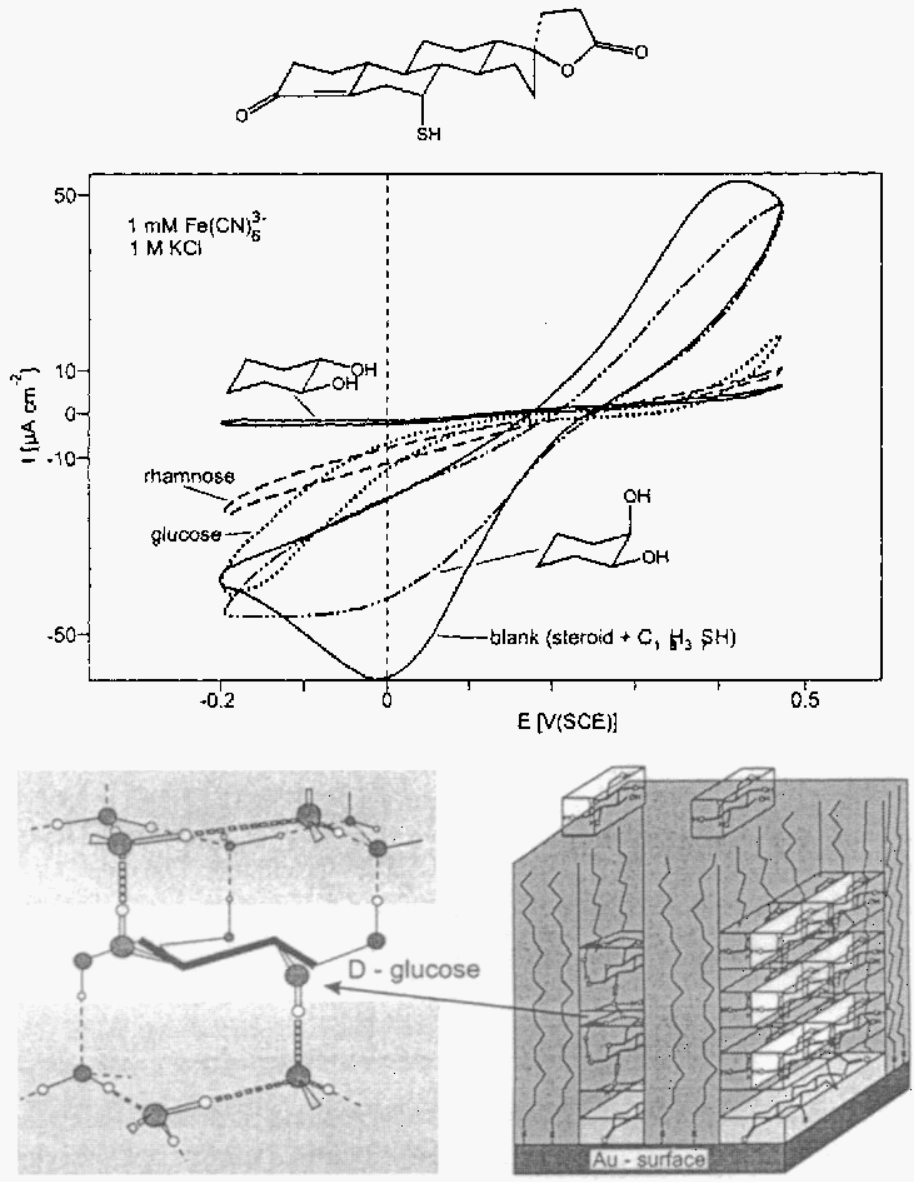

Fig. 5 Two-dimensional model of a $7 \AA$-wide steroidal hydrophobic gap filled with immobile water (not shown) or cyclohexanediol molecules. Ferricyanide transport is totally blocked as shown by cyclovoltammetry. 


\section{DIAMIDE MONOLAYERS WITH PORPHYRIN-BOTTOMED GAPS}

The octacarboxylate porphyrin shown in Fig. 6 binds irreversibly to smooth gold subphases and still fluoresces there (ref. 20). With a $\beta$-tetrapyridinium porphyrin, it forms very stable heterodimers and the fluorescence is totally quenched. The same is true if the porphyrin is fenced in with a rigid monolayer made of an even $\alpha$-thiol- $\omega$-acetamide (Fig. 4). This means that the porphyrin finds the gap, which has about the same size as itself, that it diffuses with its counterions to the porphyrin at the membrane's gap bottom and stays there. Although this process takes only a few minutes, it is surprisingly efficient and, after about 30 min., quantitative. Each gap is occupied by the cationic porphyrin. If the electrode is soaked with a 1,2trans-cyclohexanediol solution and washed, no fluorescence quenching is observed (Fig. 6). The ice-cube is again formed within the gap, although it has been widened from about 7-8 $\AA$ in the steroid case to $\geq 20 \AA$ in the porphyrin gap. One would now need at least two cyclohexanediol or seven water molecules to cover the width of the porphyrin from wall to wall.

a)

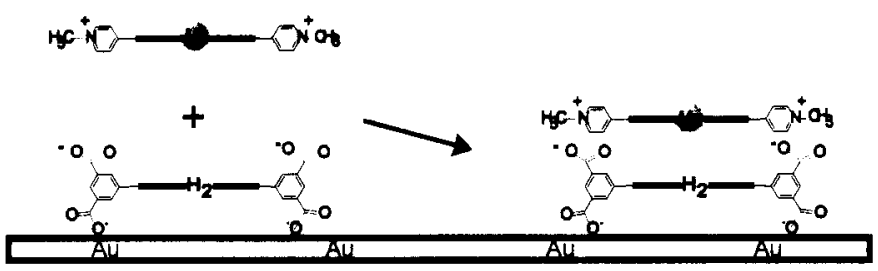

b)

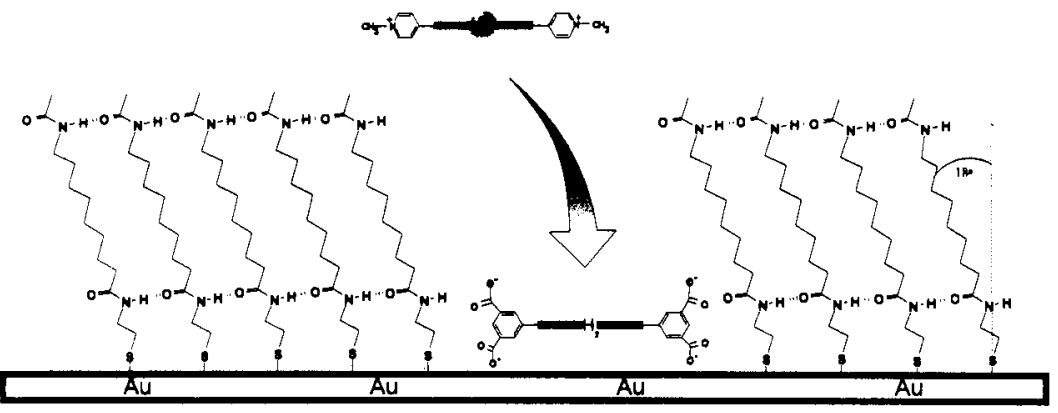

c)

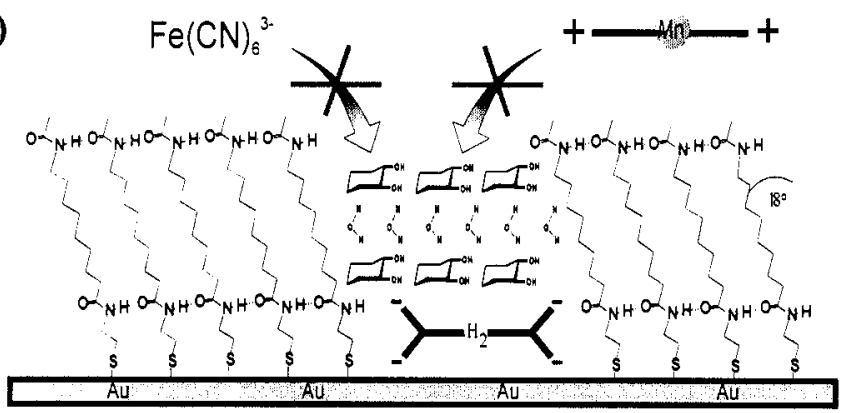

Fig. 6 (a) A porphyrin octacarboxylate binds at $\mathrm{pH} 7$ to a gold surface and fluoresces. Addition of a $10^{-5} \mathrm{M}$ solution of a manganese(III) tetra- $\beta$-pyridinium porphyrinate leads to quantitative fluorescence quenching within a few seconds. (b) If the anionic porphyrin is enclosed by a rigid diamide fence (see Fig. 4 ), the same quantitative quenching effect occurs after about $30 \mathrm{~min}$. (c) No quenching at all and no transport of ferricyanide is observed, if the membrane was soaked previously with $1 \mathrm{M}$ trans-1,2 cyclohexanediol (compare Fig. 2 ). 


\section{OUTLOOK}

The rigid diamide-membranes not only function as receptors but also provide the unique opportunity to introduce water-soluble side chains at the walls. Bolaamphiphiles with a trans-configured $\mathrm{C}=\mathrm{C}$ double bond, for example, form the same crystalline monolayers as the saturated amphiphiles. Sharpless hydroxylation with osmium(VIII) hydroxides would just oxidize the molecules which are accessible from the water-filled gaps. A circle of dihydroxyl units would thus be introduced within the gap and it should be possible to fix a second porphyrin or redox-active molecule of similar size at this site. Two redox partners, one of them photoactive, could thus be localized at a well-defined distance. The ice-like water volume in between could be doped with phenol-type transfer agents.

Another important development concerns the transfer of the membrane-gap systems from electrode to colloid surfaces, in order to obtain useful quantities. In this field, Mallouk has been groundbreaking here with his multiple layers on carb-o-sil ${ }^{\circledR}$ surfaces (ref. 23). With respect to forming stable gaps in bixin-band membranes, we have concentrated on rigid monolayers made of monomeric bixin derivatives and their mixtures with more flexible amphiphiles. Light-induced polymerization of the preformed monolayers may then lead to rigidity of domain borders.

\section{ACKNOWLEDGEMENT}

This work was supported by the European Commission within the TMR network "Artificial Photosynthesis", the Deutsche Forschungsgemeinschaft (SFB 312 "Vectorial Membrane Processes" and SFB 348 "Mesoscopic Systems") and the FNK of the Free University Berlin.

\section{REFERENCES}

1. J.-H. Fuhrhop, J. Mathieu. J. Chem. Soc., Chem. Commun., 144 (1983).

2. J.-H. Fuhrhop, D. Fritsch, B. Tesche, H. Schmiady. J. Am. Chem. Soc. 106, 1998 (1984).

3 P.E. Laibinis, G.M. Whitesides. J. Am. Chem. Soc. 114, 1990 (1992).

4. J.-H. Fuhrhop, D. Fritsch. Acc. Chem. Res. 19, 130 (1986).

5. J.-H. Fuhrhop, H. Hungerbühler, U. Siggel. Langmuir 6, 1295 (1990).

6. R.C. Zeigler, G.E. Maciel. J. Am. Chem. Soc. 113, 6349 (1991.

7. J. Israelachvili. Intermolecular and Surface Forces, p. 378ff, Academic Press, London (1992)

8. J.-H. Fuhrhop, J. Köning. Molecular Assemblies and Membranes, Monographs in Supramolecular Chemistry, .Royal Soc. Chem. (J.F. Stoddart, ed), London, i-xiii (1994)

9. G. Decker, in J.-P. Sauvage and M.W. Hosseini (eds), Comprehensive Supramolecular Chemistry, Vol. 9, Pergamon, p. 511ff, (1996).

10. J.-H. Fuhrhop, C. Demoulin, C. Böttcher, J. Köning, U. Siggel. J. Am. Chem. Soc. 114, 4159 (1992).

11. J.-H. Fuhrhop, U. Bindig, U. Siggel. J. Am. Chem. Soc. 115, 11036 (1993).

12. J.-H. Fuhrhop, D. Spiroski, C. Böttcher. J. Am. Chem. Soc. 115, 1600 (1993).

13. C. Endisch, C. Böttcher, J.-H. Fuhrhop. J. Am. Chem. Soc. 117, 8273 (1995).

14. J.-H. Fuhrhop, M. Krull, A. Schulz, D. Möbius. Langmuir 6, 497 (1990).

15. T. Komatsu, E. Tsuchida, C. Böttcher, D. Donner, C. Messerschmidt, U. Siggel, W. Stocker, J.P. Rabe, J.-H. Fuhrhop. J. Am. Chem. Soc. 119, 11660 (1997).

16. C. Messerschmidt, unpublished.

17. P. Böhme, H.-G. Hicke, C. Böttcher, J.-H. Fuhrhop. J. Am. Chem. Soc. 117, 5824 (1995).

18. J. Schneider, M. Gnade, C. Messerschmidt, J.-H. Fuhrhop., submitted for publication.

19. J.-H. Fuhrhop, T. Bedurke, M. Gnade, J. Schneider, K. Doblhofer. Langmuir 13, 455 (1997).

20. J. Zimmermann, J.-H. Fuhrhop, to be published.

21. L. Ruhlmann, J. Zimmermann, J.-H. Fuhrhop, submitted for publication.

22. L. Wang, K.B. Sharpless. J. Am. Chem. Soc.114, 7568 (1992), (see also Chem. Rev. 94, 2483 (1994).)

23. H.-G. Hong, D.D. Sachett, T.E. Mallouk. Chem. Mater. 3, 521 (1991). 